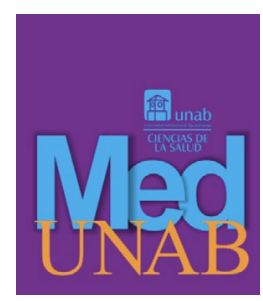

REVISTA DE LA FACULTAD

DE CIENCIAS DE LA SALUD

\title{
Nueva clasificación molecular del carcinoma de endometrio: impacto en el diagnóstico histopatológico, tratamiento y pronóstico
}

New molecular classification of endometrial carcinoma: impact on histopathological diagnosis, treatment, and prognosis

Nova classificação molecular do carcinoma de endométrio: impacto no diagnóstico histopatológico, tratamento e prognóstico

\section{Camilo Andrés Pérez-Montiel, MD. ${ }^{1}$ (D)}

1. Médico, Residente de tercer año de Patología, Universidad de Cartagena. Cartagena de Indias, Bolívar, Colombia.

Correspondencia. Camilo Andrés Pérez Montiel. Transversal 50 \# 30G-48. Apartamento 301. Barrio el Cairo. Cartagena de Indias, Bolívar, Colombia. Email. capm2908@gmail.com

\section{INFORMACIÓN DEL ARTÍCULO:}

Articulo recibido: 19 de octubre de 2020

Articulo aceptado: 20 de octubre de 2021

doi: https://doi.org/10.29375/01237047.4015

Cómo citar. Pérez-Montiel CA. Nueva clasificación molecular del carcinoma de endometrio: impacto en el diagnóstico histopatológico, tratamiento y pronóstico. MedUNAB [Internet]. 2021;24(3):365-374. doi: https://doi. org/10.29375/01237047.4015

\section{RESUMEN}

Introducción. El carcinoma de endometrio es una patología heterogénea a nivel patogénico, histopatológico y molecular. En los últimos años se han sumado esfuerzos para esclarecer y aumentar el conocimiento de las bases moleculares, logrando así dividir las pacientes en cuatro subgrupos descritos por el Atlas del Genoma del Cáncer (TCGA, por sus siglas en inglés), obteniéndose valiosa información que afecta el diagnóstico, tratamiento y pronóstico de las pacientes con esta enfermedad. El objetivo de la siguiente revisión es exponer la nueva clasificación molecular del carcinoma de endometrio, así como discutir las ventajas que esta trae a la hora de estratificar a las pacientes y tomar decisiones terapéuticas. División de los temas tratados. Se realizó una búsqueda bibliográfica no sistemática en las bases de datos PubMed, Cochrane y Medline desde el año 2014 hasta el 2020 sobre el carcinoma de endometrio y su clasificación molecular. Se expone de manera concreta y actualizada el contexto histórico, los diferentes subgrupos moleculares y cómo estos impactan en el manejo de las pacientes. Conclusiones. El carcinoma de endometrio es una 
enfermedad heterogénea a nivel histopatológico, clínico y molecular. Con la nueva clasificación y los estudios prospectivos se podrán crear nuevas estrategias que permitan brindar mejores protocolos diagnósticos y terapéuticos.

Palabras Claves:

Carcinoma; Endometrio; Neoplasias Endometriales; Inmunohistoquímica; Pronóstico; Genoma.

\begin{abstract}
Introduction. Endometrial carcinoma is a heterogeneous pathology in pathologenic, histopathological, and molecular terms. Over the last years, efforts have been made to clarify and increase knowledge of molecular bases, as such dividing patients into four subgroups described by the The Cancer Genome Atlas (TCGA), obtaining valuable information that affects the diagnosis, treatment, and prognosis of patients with this disease. The objective of this review is to exhibit the new molecular classification of endometrial carcinoma, and to discuss its advantages when stratifying patients and making therapeutic decisions. Division of Covered Topics. A non-systematic bibliographical search was carried out in the PubMed, Cochrane, and Medline databases from 2014 to 2020, on endometrial carcinoma and its molecular classification. The historical context, different molecular subgroups and how these impact patient handling are shown in a concrete and updated way. Conclusions. Endometrial carcinoma is a heterogeneous disease in histopathological, clinical, and molecular terms. With the new classification and the prospective studies, new strategies can be created to provide better diagnostic and therapeutic protocols.
\end{abstract}

Keywords:

Carcinoma; Endometrium; Endometrial Neoplasms; Immunohistochemistry; Prognosis; Genome.

\title{
RESUMO
}

Introdução. $\mathrm{O}$ carcinoma de endométrio é uma patologia heterogênea no nível patogênico, histopatológico e molecular. Nos últimos anos, foram feitos esforços para esclarecer e aumentar o conhecimento das bases moleculares, conseguindo dividir as pacientes em quatro subgrupos descritos pelo Atlas do Genoma do Câncer (TCGA, por suas siglas em inglês), obtendo informações valiosas que afetam o diagnóstico, o tratamento e o prognóstico das pacientes com esta doença. O objetivo da seguinte revisão é apresentar a nova classificação molecular do carcinoma de endométrio, bem como discutir as vantagens que ela traz no momento de estratificar as pacientes e tomar decisões terapêuticas. Divisão dos tópicos abordados. Uma pesquisa bibliográfica não sistemática foi realizada nas bases de dados PubMed, Cochrane e Medline de 2014 a 2020 sobre o carcinoma de endométrio e sua classificação molecular. São apresentados de forma concreta e atualizada o contexto histórico, os diferentes subgrupos moleculares e como esses têm impacto no tratamento das pacientes. Conclusões. O carcinoma de endométrio é uma doença heterogênea no nível histopatológico, clínico e molecular. Com a nova classificação e estudos prospectivos, novas estratégias podem ser desenvolvidas para fornecer melhores protocolos diagnósticos e terapêuticos.

Palavras-chave:

Carcinoma; Endométrio; Neoplasias do Endométrio; Imuno-Histoquímica; Prognóstico; Genoma.

\section{Introducción}

La manera en la que se aborda clínicamente a un paciente con cáncer ha cambiado en las últimas décadas. Históricamente el diagnóstico y caracterización de las neoplasias se hacía por sitio anatómico. Posteriormente, se realizaban por su subtipo histopatológico y en la actualidad se requiere de las propiedades moleculares, lo que se conoce como medicina de precisión (1).
A nivel mundial, el cáncer de endometrio ocupa el quinto lugar respecto a todos los tipos de cáncer en la población femenina, representando el $4.8 \%$ en este grupo, con 320,000 casos nuevos diagnosticados anualmente. En el mundo occidental el cáncer de endometrio se ubica en el tercer lugar y corresponde al $6-9 \%$ de todas las neoplasias malignas en las mujeres. Esta patología tiene una mortalidad de 2.1 por 100,000 mujeres/año, ocupando el puesto catorce en términos de mortalidad (2). En Latinoamérica la incidencia es del 3.5\% y tiene una 
mortalidad del $2 \%$, siendo el tercer cáncer ginecológico más frecuente (3).

En Colombia el cáncer de endometrio ocupa el tercer lugar en todos los cánceres ginecológicos, con una incidencia de 8.1 y una mortalidad de 1.7 por 100,000 mujeres/año. La prevalencia estimada de esta patología en Colombia a 1,3 y 5 años según GLOBOCAN 2020 es del 7.8, 21.3 y 32.4 por 100,000 mujeres/año, respectivamente (4).

El carcinoma de endometrio (CE) tiene una clasificación patogenética, histopatológica y molecular. La clasificación histopatológica del CE presenta una alta variabilidad interobservador y muchas veces no se correlaciona con el pronóstico de las pacientes, lo que genera imprecisiones tanto en la estratificación de riesgo como en la terapéutica (2). A pesar de que el CE es una neoplasia bastante común, la investigación molecular se había visto retrasada en comparación con otros tipos de neoplasias ginecológicas. Lo anterior podría tener explicación en que el $75 \%$ de los CE se diagnostican en estadios tempranos (I y II) y a que sus tasas de supervivencia global a 5 años son del 75$90 \%$ (5). No obstante, para las mujeres con diagnósticos en estadios avanzados o para aquellas con recurrencia, las tasas de respuesta a la quimioterapia convencional son bajas, oscilando entre el $21-36 \%$ (6).

En los últimos años se ha impulsado la investigación en el CE debido a que ha habido un aumento en la incidencia de esta patología, sobre todo en los países desarrollados (7). El objetivo del siguiente manuscrito es exponer el nuevo marco genómico utilizado para clasificar los $\mathrm{CE}$ a nivel molecular, así como describir la valiosa información que ofrece esta nueva clasificación en cuanto al pronóstico, predicción y el impacto que tiene en el diagnóstico histopatológico, investigación científica y en el manejo de las pacientes.

\section{División de los temas tratados}

\section{Metodología de búsqueda}

Se realizó una búsqueda en Medline, Science Direct y Cochrane de artículos originales y de revisión publicados desde el año 2014 hasta el 2020 en idioma inglés y español. Se utilizaron términos "endometrial carcinoma" [MeSH] OR "endometrial neoplasms" [MeSH] OR "immunohistochemistry" [MeSH] OR "prognosis" [MeSH] OR "molecular classification" [MeSH] AND "genome" para la búsqueda. Los criterios de inclusión fueron artículos originales y de revisión de los últimos 6 años que en el título y abstract describieran la clasificación molecular del carcinoma de endometrio y la relación de esta con el diagnóstico histopatológico, tratamiento y pronóstico. Se excluyeron los artículos con idioma diferente al inglés o español, artículos duplicados, estudios en animales, con contenido insuficiente o los que no se relacionaran con el objetivo de esta revisión.

La búsqueda arrojó 2,959 artículos. Posteriormente se aplicaron los criterios de inclusión y exclusión, se procedió a seleccionar los artículos más pertinentes según el resumen. Se eligieron 100 artículos, de los cuales finalmente 49 fueron escogidos para la realización del siguiente manuscrito (Figura 1).

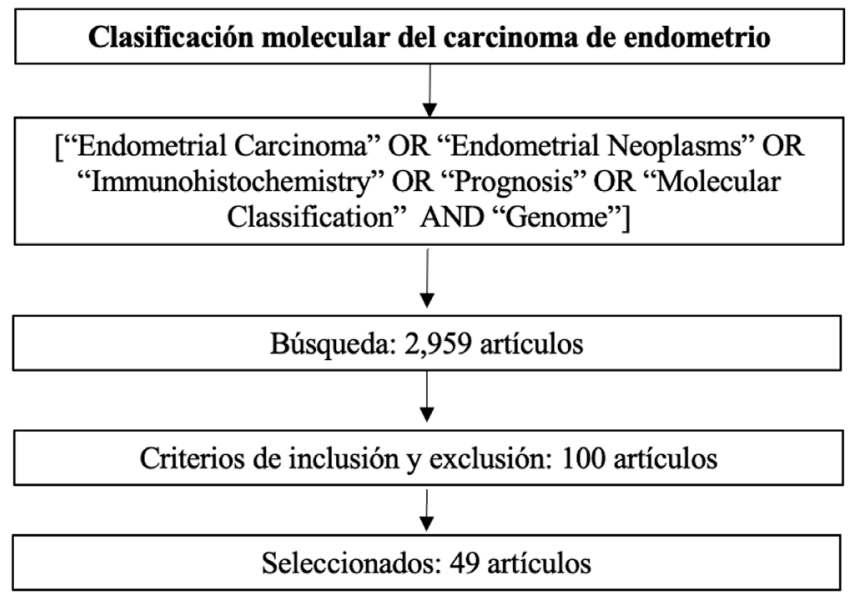

Figura 1. Diagrama de metodología de búsqueda.

Fuente: elaborado por el autor.

\section{Contexto histórico}

Fue Bokham quien en 1983 planteó la clasificación patogenética bajo la hipótesis de que había dos grandes grupos de CE (8). El tipo I son los más frecuentes (70$80 \%$ ) y están relacionados con un estímulo estrogénico persistente sin oposición. Generalmente son pacientes obesas con carcinomas bien a moderadamente diferenciados, su subtipo clásico es el carcinoma endometrioide (CAE) y tienen un pronóstico favorable. Por el contrario, el tipo II (20-30\%) son los de peor pronóstico, no se asocian a estímulo estrogénico persistente, son frecuentes en pacientes de edad avanzada y su subtipo clásico es el carcinoma seroso (CS). Esta clasificación taxonómica ha sido importante para comprender los mecanismos fisiopatológicos del CE, sin embargo, nunca ha sido utilizada en los sistemas de estratificación de riesgo y se reconoce actualmente una alta heterogeneidad entre los dos grupos (9) (Tabla 1). Además de la anterior, existe la clasificación histopatológica y la clasificación molecular del CE que se revisarán a continuación. 
Tabla 1. Clasificación patogenética del carcinoma de endometrio

\begin{tabular}{cc|}
\hline \multicolumn{2}{c|}{ Grupos de carcinoma endometrial según Bokham } \\
Tipo I & Tipo II \\
\hline Frecuencia: $70-80 \%$. & Frecuencia: $20-30 \%$. \\
$\begin{array}{c}\text { Subtipo histológico: } \\
\text { carcinoma endometrioide } \\
\text { y mucinoso. }\end{array}$ & $\begin{array}{c}\text { Subtipo histológico: } \\
\text { carcinoma seroso, } \\
\text { carcinoma de células claras, } \\
\text { carcinoma indiferenciado y } \\
\text { carcinosarcoma. }\end{array}$
\end{tabular}

\section{Factores de riesgo:} estímulo estrogénico persistente, obesidad, síndrome de ovario poliquístico.

Factores de riesgo: diabetes, hipertensión, infertilidad, síndrome de Muir - Torre, síndrome de Turner.

Alteraciones genéticas principales: PTEN, KRAS y PAX2.

\section{Alteraciones genéticas principales: TP53.}

Lesión precursora: neoplasia intraepitelial Lesión precursora: neoplasia endometrioide/hiperplasia intraepitelial serosa.

endometrial con atipia.

Descripción de las características principales según la clasificación patogenética de Bokham.

Fuente: elelaborada por el autor.

\section{Clasificación histológica y sistemas de estratificación}

Según la quinta edición de la Clasificación de Tumores del Tracto Genital Femenino de la Organización Mundial de la Salud (OMS), publicada en el 2020, los CE se clasifican en carcinoma endometrioide, carcinoma seroso, carcinoma de células claras, carcinoma indiferenciado, carcinoma des diferenciado, carcinoma mixto, carcinosarcoma y otros carcinomas endometriales (Tabla 2) (10). Clásicamente los CAE se clasifican de acuerdo a las características nucleares y arquitecturales, definiéndose tres grados histológicos según la Federación Internacional de Ginecólogos y Obstetras (FIGO) (11). Para los demás tipos de CE, se deben evaluar otros criterios morfológicos, y en los carcinomas de alto grado se utilizan frecuentemente tinciones de inmunohistoquímica para realizar los diferentes diagnósticos diferenciales. No obstante, se ha descrito una alta variabilidad interobservador a la hora de asignar un grado FIGO a los CAE (Kappa 0.41-0.68) o al realizar los diagnósticos diferenciales entre los carcinomas de alto grado (Ejemplo: CAE FIGO 3 vs. CS) (12). Sumado a lo anterior, puede existir discordancia entre el diagnóstico que se da en el legrado endometrial a comparación del que se designa con el espécimen quirúrgico (13). Por lo cual la clasificación histológica presenta desventajas a la hora de encasillar a las pacientes en grupos de riesgo.

Tabla 2. Clasificación histológica de los carcinomas de endometrio según la OMS (2020).

Carcinoma endometrioide Carcinoma seroso

Carcinoma de células claras

Carcinoma indiferenciado

Carcinoma desdiferenciado

Carcinoma mixto

Carcinosarcoma

Otros carcinomas endometriales

Fuente: World Health Organization. Publication of the WHO classification of tumours, 5th Edition, Volume 4: Female Genital Tumours [Internet]. Who.int. [consultado el 27 de abril de 2021]. Recuperado a partir de https://www.iarc.who.int/ news-events/publication-of-the-who-classification-of-tumours5th-edition-volume-4-female-genital-tumours/. (10)

A lo largo de los años, se han desarrollado diferentes modelos de estratificación de riesgo para determinar la probabilidad de recurrencia y guiar el tratamiento adyuvante de las pacientes con CE. Todos los modelos que existen en la actualidad incorporan parámetros patológicos como el subtipo histológico, el grado y el estadio (14). Como se mencionó previamente, existe una alta variabilidad interobservador para definir tanto el tipo histológico como el grado; por ende, esto afecta la confiabilidad y la reproducibilidad de los modelos de estratificación de riesgo, pudiendo conllevar a tratamientos muy agresivos o insuficientes (1). Existen cinco sistemas de estratificación de riesgo para el CE, de los cuales el Sistema de Clasificación Europeo Modificado de Médicos Oncólogos (ESMO) es el que tiene mayor evidencia a la hora de predecir la recurrencia y las metástasis ganglionares en los estadios tempranos, dividiendo a las pacientes en riesgo bajo (recurrencia 2-10\%), riesgo intermedio (recurrencia $20-25 \%$ ) y riesgo alto (recurrencia $30-65 \%$ ) (15). Se considera que los sistemas actuales no son precisos (1). Igualmente, la estratificación se hace con base en el espécimen quirúrgico, lo que refleja la necesidad de tener información más temprana para dirigir de una mejor manera el tratamiento. En vista de todos los argumentos previamente discutidos, surge la necesidad de integrar las características moleculares con el fin 
de predecir mejor el pronóstico de las pacientes e implementar mejores conductas terapéuticas (16).

\section{Clasificación molecular del carcinoma de endometrio}

El Atlas del Genoma del Cáncer(TCGA) realizó el análisis molecular más completo de la actualidad para evaluar los componentes moleculares del CE. Este comprendió el estudio de la secuenciación del genoma, la secuenciación del Exoma, ensayos de Inestabilidad microsatelital (MSI) y el análisis del número de copias somáticas (17). Se estudiaron $232 \mathrm{CE}$ y de acuerdo a los resultados se clasificaron en cuatro subgrupos: UltramutadoPolimerasa Épsilon (POLE), Hipermutado-Inestabilidad Microsatélite, Bajo Número de Copias y Alto Número de Copias. Cada subgrupo presenta diferentes aberraciones genómicas y se correlaciona con la supervivencia (18) (Tabla 3).

Tabla 3. Resumen de las principales características de los subgrupos moleculares del carcinoma de endometrio según el Atlas del Genoma del Cáncer (TCGA).

\begin{tabular}{|c|c|}
\hline $\begin{array}{l}\text { Clasificación } \\
\text { molecular }\end{array}$ & Características más importantes \\
\hline $\begin{array}{c}\text { Ultramutado } \\
\text { - Polimerasa } \\
\text { Épsilon } \\
\text { (POLE). }\end{array}$ & $\begin{array}{l}\text { - Alto número de mutaciones y mutación } \\
\text { puntual de polimerasa épsilon. } \\
\text { - Frecuentemente adenocarcinomas } \\
\text { endometrioides de alto grado. } \\
\text { - Patrón de invasión frontal y superficial } \\
\text { del miometrio. } \\
\text { - Morfología ambigua. } \\
\text { - Infiltrado linfocítico. }\end{array}$ \\
\hline $\begin{array}{l}\text { Hipermutado } \\
\text { - Inestabilidad } \\
\text { Microsatélite. }\end{array}$ & $\begin{array}{l}\text { - Inestabilidad microsatelital, } \\
\text { principalmente por metilación MLH1. } \\
\text { - Frecuentemente adenocarcinomas } \\
\text { endometrioides. } \\
\text { - Infiltrado linfocítico. } \\
\text { - Patrón de invasión con glándulas } \\
\text { elongadas, microquísticas o } \\
\text { multifragmentadas. } \\
\text { - Mayor probabilidad de invasión } \\
\text { linfovascular. }\end{array}$ \\
\hline $\begin{array}{l}\text { Bajo Número } \\
\text { de Copias }\end{array}$ & $\begin{array}{l}\text { - Estable a nivel genético. } \\
\text { - No tiene errores en las proteínas de } \\
\text { reparación del ADN. } \\
\text { - Frecuentemente adenocarcinomas } \\
\text { endometrioides con positividad para } \\
\text { receptores de estrógenos y progesterona. }\end{array}$ \\
\hline $\begin{array}{l}\text { Alto Número } \\
\text { de Copias }\end{array}$ & $\begin{array}{l}\text { - Mutación TP53. } \\
\text { - Alta variabilidad en el número de copias. } \\
\text { - Frecuentemente son carcinomas serosos. }\end{array}$ \\
\hline
\end{tabular}

Descripción de las características principales según la clasificación patogenética de Bokham.

Fuente: elelaborada por el autor.
El subgrupo POLE obtuvo resultados favorables incluso dentro de los carcinomas de alto grado (19). Este subgrupo se caracterizó por presentar mutaciones en el dominio de la Exonucleasa épsilon, el cual es un gen que participa en la replicación y la reparación del ADN (20). También se observó un elevado porcentaje de transversiones $\mathrm{C}>\mathrm{A}$ y un bajo porcentaje de transversiones $\mathrm{C}>\mathrm{G}(1,18)$. Estos tumores presentaron un alto número de mutaciones somáticas que superaban las 100 mutaciones por Megabase $(\mathrm{Mb})$ en la mayoría de los casos (21). Posteriormente, otras investigaciones demostraron que a nivel histopatológico los $\mathrm{CE}$ del subgrupo POLE se distinguían por ser CAE de alto grado, con un patrón de invasión frontal y superficial del miometrio, con presencia de células gigantes tumorales e infiltrado linfocitario prominente intraepitelial (22) (Figura 2). Los carcinomas que presentan mayor dificultad a la hora de su evaluación histológica son los pertenecientes al subgrupo POLE debido a su morfología ambigua (23).

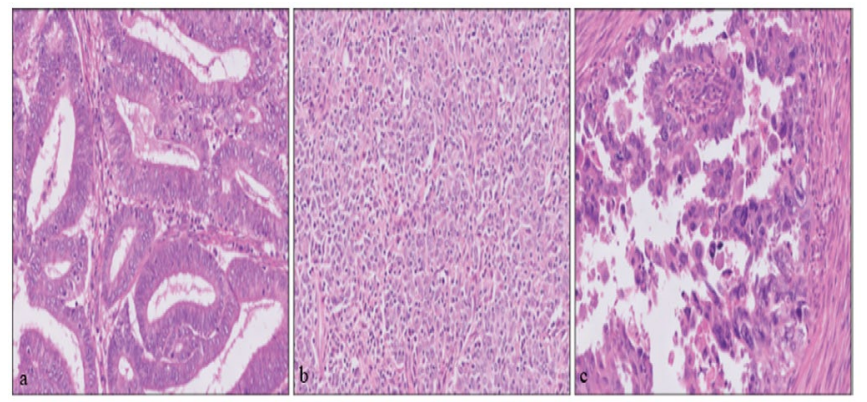

a. Adenocarcinoma de endometrio FIGO grado 1. b. Adenocarcinoma endometrioide FIGO grado 3 con un prominente infiltrado linfocítico intratumoral (TILs). c. Carcinoma de endometrio de alto grado, de morfología ambigua, con alto pleomorfismo nuclear y células gigantes tumorales.

Figura 2: Características histopatológicas principales en los carcinomas de endometrio del subgrupo POLE.

Fuente: McAlpine J, Leon-Castillo A, Bosse T. The rise of a novel classification system for endometrial carcinoma; integration of molecular subclasses. J Pathol [Internet]. 2018;244(5):538-549. doi: https://doi.org/10.1002/path.5034 (18)

El siguiente subgrupo se denomina HipermutadoInestabilidad microsatélite (MSI, por sus siglas en inglés). La inestabilidad microsatelital es secundaria a errores en las proteínas que participan en la reparación del ADN postreplicativo (24). Estas proteínas son: MLH1, PMS2, MSH2 y MSH6. El mecanismo subyacente principal que se identificó fue el silenciamiento epigenético de MLH1 por hipermetilación (25). Hay pacientes con $\mathrm{CE}$ en las cuales los errores en las proteínas de reparación del ADN están causados por mutaciones somáticas o de la línea germinal, lo que las predispone a padecer Síndrome de Lynch, caracterizado por un 
alto riesgo de sufrir carcinoma colorrectal y CE (26). Histopatológicamente la mayoría de los carcinomas en este subgrupo son endometrioides, aunque se han descrito subtipos no endometrioides (27). Similar a los del subgrupo POLE, estos carcinomas presentan linfocitos intraepiteliales asociados a un patrón de infiltración característico de glándulas elongadas, microquísticas o multifragmentadas (MELF, por sus siglas en inglés) con una mayor probabilidad de tener invasión linfovascular (28).

El tercer subgrupo molecular se denominó Bajo Número de Copias, se distinguió porque era relativamente estable a nivel genético, no tenía errores en las proteínas de reparación del $\mathrm{ADN}$ y tenía un número moderado de mutaciones, sobre todo en las vías de señalización PI3K/Akt y Wnt (27). A nivel histopatológico estaba compuesto casi que exclusivamente por CAE con positividad para los receptores de estrógeno y progesterona (29).

Finalmente, el cuarto grupo tiene por nombre Alto Número de Copias. Genéticamente son similares a los carcinomas serosos de alto grado del ovario que presentan una alta variabilidad en el número de copias, con mutaciones frecuentes en TP53 (92\%). Histopatológicamente, la mayoría son CS, sin embargo, llamó la atención que el $26 \%$ de los CAE se encuentran en este grupo, lo que motivó a que se le conozcan en la práctica como "carcinomas endometrioides semejantes a carcinomas serosos" (30).

\section{Pronóstico y aplicabilidad clínica}

Los CS se encuentran en el subgrupo de alto número de copias, sin embargo, los CAE se distribuyen en los cuatro subgrupos de la siguiente manera: en el subgrupo POLE, el $6.4 \%$ de los CAE FIGO grado 1 y 2 y el $17.3 \%$ de los CAE FIGO grado 3. En el subgrupo inestabilidad microsatélite, el $28.6 \%$ de los CAE FIGO grado 1 y 2 , y el $54 \%$ de los CAE FIGO grado 3. En el subgrupo con bajo número de copias, el $60 \%$ de los CAE FIGO grado 1 y 2 , y el $8.7 \%$ de los CAE FIGO grado 3. Finalmente, en el subgrupo con alto número de copias el $5 \%$ de los CAE FIGO grado 1 y 2 , y el $19.6 \%$ de los CAE FIGO grado $3(30,31)$ (Tabla 4). Según su comportamiento biológico, el subgrupo con el mejor pronóstico es el POLE y el que tiene el peor pronóstico es el de alto número de copias (32).

Una vez salió a la luz científica la nueva clasificación molecular propuesta por el TCGA, se hicieron evidentes varias desventajas: la metodología con la que se realizaron estos estudios era bastante compleja, costosa, sofisticada y poco reproducible para realizarse en los laboratorios convencionales a nivel mundial. Además, la clasificación fue el resultado del estudio de una cohorte pequeña y con alta variabilidad clínica, motivo por el cual no tenía el poder estadístico suficiente (33). Debido a esto, varios grupos a nivel mundial emprendieron la tarea de encontrar la forma de hacer pruebas de manera rutinaria en tejidos fijados con formol e incluidos en bloques de parafina, con el fin de obtener mayor aplicabilidad clínica y que sean similares a la clasificación molecular propuesta por el TCGA (1).

Tabla 4. Distribuciones del carcinoma endometrioide y el carcinoma seroso en los diferentes subgrupos moleculares del carcinoma de endometrio.

\begin{tabular}{|c|c|}
\hline $\begin{array}{l}\text { Clasificación } \\
\text { molecular }\end{array}$ & Características más importantes \\
\hline $\begin{array}{l}\text { Ultramutado } \\
\text { - Polimerasa } \\
\text { Épsilon } \\
\text { (POLE). }\end{array}$ & $\begin{array}{l}\text { - Carcinomas endometrioides FIGO } \\
\text { grado } 1 \text { y } 2: 6.4 \% \text {. } \\
\text { - Carcinomas endometrioides FIGO } \\
\text { grado 3: } 17.3 \% \text {. }\end{array}$ \\
\hline $\begin{array}{l}\text { Hipermutado } \\
\text { - Inestabilidad } \\
\text { Microsatélite }\end{array}$ & $\begin{array}{l}\text { - Carcinomas endometrioides FIGO } \\
\text { grado } 1 \text { y } 2: 28.6 \% \text {. } \\
\text { - Carcinomas endometrioides FIGO } \\
\text { grado 3: } 54 \% \text {. }\end{array}$ \\
\hline $\begin{array}{l}\text { Bajo Número } \\
\text { de Copias }\end{array}$ & $\begin{array}{l}\text { - Carcinomas endometrioides FIGO grado } \\
1 \text { y } 2: 60 \% \text {. } \\
\text { - Carcinomas endometrioides FIGO grado } \\
\text { 3: } 8.7 \% \text {. }\end{array}$ \\
\hline $\begin{array}{l}\text { Alto Número } \\
\text { de Copias }\end{array}$ & $\begin{array}{l}\text { - Carcinomas serosos. } \\
\text { - Carcinomas endometrioides FIGO grado } \\
\text { 1 y 2: } 5 \% \text {. } \\
\text { - Carcinomas endometrioides FIGO grado } \\
\text { 3: } 19.6 \% \text {. }\end{array}$ \\
\hline
\end{tabular}

Fuente: elelaborada por el autor.

El grupo de Vancouver propuso un método de clasificación que se denomina ProMisE (Proactive Molecular Risk Classifier for Endometrial Cancer), en este estudio se incluyeron carcinomas endometrioides, carcinomas serosos, mixtos e indiferenciados (34). Mediante pruebas de inmunohistoquímica se evaluó inestabilidad microsatelital para MLH1, MSH2, MSH6, PMS2 y disfunción en la reparación del ADN, posteriormente se determinó la secuenciación para el subgrupo POLE y finalmente se hicieron pruebas de inmunohistoquímica para carcinomas con p53 
anormal y p53 nativo $(1,35)$. De esta manera, los CE se subclasificaron en cuatro subgrupos de pronóstico con base en las pruebas realizadas, los cuales reflejaron los resultados por el TCGA. En el subgrupo POLE el pronóstico fue excelente con un Hazard ratio (HR) de 0.4 para la supervivencia libre de recurrencia, mientras que el subgrupo p53 con alto número de copias tuvo un HR de $1.64(\mathrm{p}=0.024)$ en comparación con el subgrupo p53 nativo con bajo número de copias (36).

El grupo de Stello y colaboradores realizó una prueba que clasifica los CE en los cuatro subgrupos ya discutidos previamente. Con esta prueba se evidenció que los subgrupos POLE y de inestabilidad microsatélite fueron los que tuvieron mejor pronóstico (37). Posteriormente se hizo un estudio en el cual se siguió una cohorte de 834 pacientes provenientes de los ensayos clínicos PORTEC - 1 y PORTEC - 2, en la cual se concluyó que al tomar en cuenta los cuatro subgrupos moleculares sumado a la invasión linfovascular mejoraba la estratificación del riesgo de cada paciente cuando se comparaba con los grupos pronósticos que se hacían con características clínico-patológicas (38).

\section{Impacto de la clasificación molecular en la terapéutica}

Integrar la información clínico-patológica junto con la clasificación molecular podría brindar nuevas directrices al momento de abordar a las pacientes con $\mathrm{CE}$, tanto en la valoración preoperatoria, en el tratamiento adyuvante, en la vigilancia y en la enfermedad recurrente. En el ámbito preoperatorio se espera que a todas las pacientes con $\mathrm{CE}$ se les realice la clasificación molecular. Actualmente ante un diagnóstico de carcinoma de alto grado, se recomienda además de la resección quirúrgica, la linfadenectomía, independientemente del resultado de la clasificación molecular. Sin embargo, dado que las pacientes en el subgrupo POLE tienen muy baja probabilidad de tener enfermedad diseminada más allá del útero así tengan un carcinoma de alto grado, solo está recomendada la Histerectomía simple más Salpingooforectomía bilateral (17). Todas las pacientes con p53 anormal requerirían un tratamiento quirúrgico agresivo que incluye resección de ganglios linfáticos y omentectomía debido al pobre pronóstico que tiene este subgrupo. Se han evaluado otros escenarios en los cuales la clasificación molecular sería de gran ayuda, como por ejemplo las pacientes jóvenes. No obstante, se requieren más estudios prospectivos que demuestran la eficacia de estas conductas terapéuticas $(17,39,40)$.
Las opciones de tratamiento adyuvante podrían verse afectadas con la clasificación molecular. Actualmente se está desarrollando el ensayo clínico PORTEC-4a, el cual comparará el tratamiento adyuvante estándar en pacientes con carcinoma de endometrio de riesgo intermedio y alto, contra tratamientos adyuvantes diferentes basados en el perfil molecular de cada paciente. Con este tipo de investigaciones se obtendrán resultados que podrían dirigir de una manera diferente el tratamiento adyuvante según el subgrupo molecular que presente cada paciente (41).

Con la clasificación molecular, las directrices de vigilancia en las pacientes se podrían personalizar, dado que según los subgrupos moleculares, se esperaría que las pacientes con carcinomas con p53 con alto número de copias reciban un seguimiento más estricto en comparación de las pacientes con carcinomas POLE. Sin embargo, estas determinaciones deben ser valoradas a la luz de los resultados de los ensayos clínicos que se están realizando en la actualidad (17). La clasificación molecular brindaría nuevas bases de terapia inmunológica (42). Algunos estudios han concluido que los subgrupos POLE y de inestabilidad microsatelital tienen un abundante número de neoepítopos/neoantígenos que se unen al complejo mayor de histocompatibilidad (MCH), induciendo una mejor respuesta de los linfocitos $\mathrm{T}$ CD8 antitumorales $(43,44)$. Estos carcinomas evaden la respuesta inmunológica regulando al alza moléculas de control inmunológico, como lo son PD - 1 y PDL - 1, las cuales alteran negativamente los linfocitos $\mathrm{T}$ CD8 antitumorales (45). Existen agentes terapéuticos denominados inhibidores de puntos de control, los cuales bloquean estas moléculas de control inmunológico, ocasionando una mejor respuesta y regresión tumoral $(46,47)$. Se ha evidenciado que al tratar a las pacientes del subgrupo POLE y de inestabilidad microsatelital con enfermedad metastásica usando fármacos como el Nivolumab o Pembrolizumab, hay una respuesta eficaz al tratamiento que se refleja en la disminución del tamaño del tumor metastásico, con tasas de respuesta sostenidas a los 7 y 14 meses para Nivolumab y Pembrolizumab, respectivamente $(47,48)$.

\section{Conclusiones}

La clasificación patogenética e histopatológica del CE presenta una alta variabilidad interobservador y no se correlaciona correctamente con el pronóstico. La clasificación molecular romperá paradigmas tanto en el diagnóstico como en el tratamiento, debido a que se describen 4 subgrupos biológicamente distintos, 
aportando información valiosa para el diagnóstico, tratamiento quirúrgico, tratamiento adyuvante $\mathrm{y}$ programas de seguimiento. Los estudios prospectivos que se realizan en la actualidad están dirigidos a demostrar el beneficio clínico que tendrán las pruebas moleculares en conjunción con el diagnóstico histopatológico, de esta manera se busca hallar la mejor forma de utilizar esta valiosa herramienta, permitiendo una comprensión integral de los patrones moleculares y mejorar el resultado de las pacientes con esta patología.

\section{Conflictos de intereses}

El autor declara no tener conflictos de intereses.

\section{Financiación}

Para la realización de este manuscrito, no existió ningún tipo de financiación externa al autor.

\section{Referencias}

1. Talhouk A, McAlpine JN. New classification of endometrial cancers: the development and potential applications of genomic-based classification in research and clinical care. Gynecol Oncol Res Pract [Internet]. 2016;3:14. doi: https://doi.org/10.1186/s40661-0160035-4

2. Zhang Y, Liu H, Yang S, Zhang J, Qian L, Chen X. Overweight, Obesity and Endometrial Cancer Risk: Results from a Systematic Review and Meta-Analysis. Int J Biol Markers [Internet]. 2014;29(1):e21-9. doi: https://doi.org/10.5301/JBM.5000047

3. Pardo C, Cendales R. Incidencia, mortalidad y prevalencia de cáncer en Colombia, 2007-2011, Primera edición. Bogotá. D.C. Instituto Nacional de Cancerología, 2015, v.1.p. 148.

4. Sung H, Ferlay J, Siegel RL, Laversanne M, Soerjomataram I, Jemal A, et al. Global Cancer Statistics 2020: GLOBOCAN Estimates of Incidence and Mortality Worldwide for 36 cancers in 185 Countries. CA Cancer J Clin [Internet]. 2021;(caac.21660). doi: https://doi.org/10.3322/caac. 21660

5. Morice P, Leary A, Creutzberg C, Abu-Rustum $\mathrm{N}$, Darai E. Endometrial cancer. Lancet [Internet]. 2016;387(10023):1094-108. doi: https://doi. org/10.1016/S0140-6736(15)00130-0

6. Del Carmen MG, Boruta DM, Schorge JO. Recurrent Endometrial Cancer. Clin Obstet Gynecol [Internet]. 2011 Jun; 54 (2):266-77. doi: https://doi.org/10.1097/ GRF.0b013e318218c6d1
7. Jung KW, Won YJ, Kong HJ, Oh CM, Lee DH, Lee JS. Prediction of cancer incidence and mortality in Korea, 2014. Cancer Res Treat [Internet]. 2014;46(2):124-30. doi: https://doi.org/10.4143/crt.2014.46.2.124

8. Bokhman JV. Two pathogenetic types of endometrial carcinoma. Gynecol Oncol [Internet]. 1983;15(1):107. doi: https://doi.org/10.1016/0090-8258(83)90111-7

9. Abdulfatah E, Wakeling E, Sakr S, Al-Obaidy K, Bandyopadhyay S, Morris R, et al. Molecular classification of endometrial carcinoma applied to endometrial biopsy specimens: Towards early personalized patient management. Gynecol Oncol [Internet]. 2019;154(3):467-474. doi: https://doi. org/10.1016/j.ygyno.2019.06.012

10. World Health Organization. Publication of the WHO Classification of Tumours, 5th Edition, Vollume 4: Female genital Tumours [Internet]. Who.int. [consultado el 27 de abril de 2021]. Recuperado a partir de https://www.iarc.who.int/news-events/ publication-of-the-who-classification-of-tumours5th-edition-volume-4-female-genital-tumours/

11. Murali R, Davidson B, Fadare O, Carlson JA, Crum CP, Gilks CB, et al. High-grade Endometrial Carcinomas: Morphologic and Immunohistochemical Features, Diagnostic Challenges and Recommendations. Int J Gynecol Pathol [Internet]. 2019;38:S40-S63. doi: https://doi.org/10.1097/PGP.0000000000000491

12. Han G, Soslow RA, Wethington S, Levine DA, Bogomolniy F, Clement PB, et al. Endometrial Carcinomas With Clear Cells: A Study of a Heterogeneous Group of Tumors Including Interobserver Variability, Mutation Analysis, and Immunohistochemistry with HNF-1 $\beta$. Int J Gynecol Pathol [Internet]. 2015;34(4):323-33. doi: https://doi. org/10.1097/PGP.0000000000000162

13. Mhlongo SE, NaidooTD, Makhathini BS. Discrepancy between preoperative endometrial sampling and hysterectomy diagnosis in endometrial cancer. South Afr J Gynaecol Oncol [Internet]. 2020;12(1):13-6. doi: https://doi.org/10.1080/20742835.2020.1754659

14. Imai K, Kato H, Katayama K, Nakanishi K, Kawano A, Iura A, et al. A preoperative risk-scoring system to predict lymph node metastasis in endometrial cancer and stratify patients for lymphadenectomy. Gynecol Oncol [Internet]. 2016;142(2):273-7. doi: https://doi. org/10.1016/j.ygyno.2016.06.004

15. Bendifallah S, Canlorbe $G$, Collinet $P$, Arsène E, Huguet F, Coutant C, et al. Just how accurate are the major risk stratification systems for earlystage endometrial cancer? $\mathrm{Br} \mathrm{J}$ Cancer [Internet]. 2015;112(5):793-801. doi: https://doi.org/10.1038/ bjc. 2015.35

16. McConechy MK, Talhouk A, Leung S, Chiu D, Yang W, Senz J, et al. Endometrial carcinomas 
with POLE Exonuclease Domain Mutations Have a Favorable Prognosis. Clin Cancer Res [Internet]. 2016;22(12):2865-73. doi: https://doi. org/10.1158/1078-0432.ccr-15-2233

17. Levine DA, The Cancer Genome Atlas Research Network. Integrated genomic characterization of endometrial carcinoma. Nature [Internet]. 2013;497(7447):67-73. doi: https://doi.org/10.1038/ nature12113

18. McAlpine J, Leon-Castillo A, Bosse T. The rise of a novel classification system for endometrial carcinoma; integration of molecular subclasses. J Pathol [Internet]. 2018;244:538-549. doi: https://doi. org/10.1002/path.5034

19. Rayner E, van Gool IC, Palles C, Kearsey SE, Bosse $\mathrm{T}$, Tomlinson I, et al. A panoply of errors: polymerase proofreading domain mutations in cancer. Nat Rev Cancer [Internet]. 2016;16:71-81. doi: https://doi. org/10.1038/nrc.2015.12

20. Talhouk A, McConechy MK, Leung S, Li-Chang HH, Kwon JS, Melnyk N, et al. A clinically applicable molecular-based classification for endometrial cancers. Br J Cancer [Internet]. 2015;113: 299-310. doi: https://doi.org/10.1038/bjc.2015.190

21. Shinbrot E, Henninger EE, Weinhold N, Covington KR, Göksenin AY, Schultz N, et al. Exonuclease mutations in DNA polymerase epsilon reveal replication strand specific mutation patterns and human origins of replication. Genome Res [Internet]. 2014;24:1740-50. doi: https://doi.org/10.1101/gr.174789.114

22. Eggink FA, Van Gool IC, LearyA, Pollock PM, Crosbie EJ, Mileshkin L, et al. Immunological profiling of molecularly classified high-risk endometrial cancers identifies POLE-mutant and microsatellite unstable carcinomas as candidates for checkpoint inhibition. Oncoimmunology [Internet]. 2017;6(2):e1264565. doi: https://doi.org/10.1080/2162402X.2016.1264565

23. Hussein YR, Weigelt B, Levine DA, Schoolmeester JK, Dao LN, Balzer BL, et al. Clinicopathological analysis of endometrial carcinomas harboring somatic POLE exonuclease domain mutations. Mod Pathol [Internet]. 2015;28:505-14. doi: https://doi. org/10.1038/modpathol.2014.143

24. Simpkins SB, Bocker T, Swisher EM, Mutch DG, Gersell DJ, Kovatich AJ, et al. MLH1 Promoter Methylation and Gene Silencing is the Primary Cause of Microsatellite Instability in Sporadic Endometrial Cancers. Hum Mol Genet [Internet]. 1999;8(4):661666. doi: https://doi.org/10.1093/hmg/8.4.661

25. Liccardo R, De Rosa M, Izzo P, Duraturo F. Novel Implications in Molecular Diagnosis of Lynch Syndrome. Gastroenterol Res Pract [Internet]. 2017;2017:12p. doi: https://doi. org/10.1155/2017/2595098
26. DeLair DF, Burke KA, Selenica P, Lim RS, Scott $\mathrm{SN}$, Middha S, et al. The genetic landscape of endometrial clear cell carcinomas. J Pathol [Internet]. 2017;243(2):230-241. doi: https://doi.org/10.1002/ path.4947

27. Stelloo E, Nout RA, Osse EM, JürgenliemkSchulz IJ, Jobsen JJ, Lutgens LC, et al. Improved Risk Assessment by Integrating Molecular and Clinicopathological Factors in Earlystage Endometrial Cancer-Combined Analysis of the PORTEC Cohorts. Clin Cancer Res [Internet]. 2016;22(16):4215-24. doi: https://doi. org/10.1158/1078-0432.CCR-15-2878

28. Visser NC, Bulten J, van der Wurff AA, Boss EA, Bronkhorst CM, Feijen HW, et al. PIpelle Prospective ENDOmetrial carcinoma (PIPENDO) study, preoperative recognition of high risk endometrial carcinoma: a multicentre prospective cohort study. BMC Cancer [Internet]. 2015;15:487. doi: https:// doi.org/10.1186/s12885-015-1487-3

29. Carlson J, McCluggage WG. Reclassifying endometrial carcinomas with a combined morphological and molecular approach. Curr Opin Oncol [Internet]. 2019;31(5):411-419. doi: https:// doi.org/10.1097/CCO.0000000000000560

30. Suarez AA, Felix AS, Cohn DE. Bokhman Redux: Endometrial cancer "types" in the 21st century. Gynecol Oncol [Internet]. 2017;144(2):243-249. doi: https://doi.org/10.1016/j.ygyno.2016.12.010

31. Alvarado-Cabrero I, Estevez-Castro R, ValenciaCedillo R. Estado actual del carcinoma de endometrio. Patología Rev Latinoam [Internet]. 2020;58:1-9. Recuperado a partir de: http://www. revistapatologia.com/content/2020/0106201326.pdf

32. Bosse T, Nout RA, McAlpine JN, McConechy MK, Britton H, Hussein YR, et al. Molecular Classification of Grade 3 Endometrioid Endometrial Cancers Identifies Distinct Prognostic Subgroups. Am J Surg Pathol [Internet]. 2018;42(5):561-568. doi: https:// doi.org/10.1097/PAS.0000000000001020

33. Cosgrove CM, Cohn DE, Hampel H, Frankel WL, Jones D, McElroy JP, et al. Epigenetic silencing of MLH1 in endometrial cancers is associated with larger tumor volume, increased rate of lymph node positivity and reduced recurrence-free survival. Gynecol Oncol [Internet]. 2017;146(3):588-595. doi: https://doi.org/10.1016/j.ygyno.2017.07.003

34. Hoang LN, Kinloch MA, Leo JM, Grondin K, Lee $\mathrm{CH}$, Ewanowich $\mathrm{C}$, et al. Interobserver Agreement in Endometrial Carcinoma Histotype Diagnosis Varies Depending on The Cancer Genome Atlas (TCGA)-based Molecular Subgroup. Am J Surg Pathol [Internet]. 2017;41(2):245-252. doi: https:// doi.org/10.1097/PAS.0000000000000764 
35. Talhouk A, McConechy MK, Leung S, Yang W, Lum A, Senz J, et al. Confirmation of ProMisE: A simple, genomics-based clinical classifier for endometrial cancer. Cancer [Internet]. 2017;123(5):802-813. doi: https://doi. org/10.1002/cncr.30496

36. Stelloo E, Bosse T, Nout RA, MacKay HJ, Church DN, Nijman HW, et al. Refining prognosis and identifying targetable pathways for high-risk endometrial cancer; a TransPORTEC initiative. Mod Pathol [Internet]. 2015;28:836-844. doi: https://doi.org/10.1038/ modpathol.2015.43

37. Stelloo E, Nout RA, Osse EM, Jürgenliemk-Schulz IJ, Jobsen JJ, Lutgens LC, et al. Improved Risk Assessment by Integrating Molecular and Clinicopathological Factors in Early-stage Endometrial Cancer-Combined Analysis of the PORTEC Cohorts. Clin Cancer Res [Internet]. 2016;22(16):4215-24. doi: https://doi.org/10.1158/10780432.CCR-15-2878

38. Van Esterik M, Van Gool IC, de Kroon CD, Nout RA, Creutzberg CL, Smit VTHBM, et al. Limited impact of intratumour heterogeneity on molecular risk assignment in endometrial cancer. Oncotarget [Internet]. 2017;8:25542-51. doi: https://doi.org/10.18632/ oncotarget.16067

39. Talhouk A, Hoang LN, McConechy MK, Nakonechny Q, Leo J, Cheng A, et al. Molecular classification of endometrial carcinoma on diagnostic specimens is highly concordant with final hysterectomy: Earlier prognostic information to guide treatment. Gynecol Oncol [Internet]. 2016;143(1):46-53. doi: https://doi. org/10.1016/j.ygyno.2016.07.090

40. ISRCTN registry.PORTEC-4a: Randomised Trial of Standard or Molecular Profile-Based Recommendation for Radiotherapy after Surgery for Women with Early Stage Endometrial Cancer. ISRCTN [Internet]. 2016. doi: https://doi.org/10.1186/ISRCTN11659025

41. Howitt BE, Shukla SA, Konstantinopoulos PA. Neoepitopes and CD3-Positive and CD8-Positive Cells in Polymerase e-Mutated and Microsatellite-
Instable Endometrial Cancers--Reply. JAMA Oncol [Internet]. 2016;2(1):141-2. doi: https://doi.org/10.1001/ jamaoncol.2015.3903

42. Brown SD, Warren RL, Gibb EA, Martin SD, Spinelli JJ, Nelson BH, et al. Neo-antigens predicted by tumor genome meta-analysis correlate with increased patient survival. Genome Res [Internet]. 2014;24:743-750. doi: https://doi.org/10.1101/gr.165985.113

43. Schumacher TN, Schreiber RD. Neoantigens in cancer immunotherapy. Science [Internet]. 2015;348(6230):6974. doi: https://doi.org/10.1126/science.aaa4971

44. Sharpe AH, Pauken KE. The diverse functions of the PD1 inhibitory pathway. Nat Rev Immunol [Internet]. 2018;18:153-167. doi: https://doi.org/10.1038/ nri.2017.108

45. Topalian SL, Drake CG, Pardoll DM. Immune Checkpoint Blockade: A Common Denominator Approach to Cancer Therapy. Cancer Cell [Internet]. 2015;27(4):450-61. doi: https://doi.org/10.1016/j. ccell.2015.03.001

46. Ott PA, Bang YJ, Berton-Rigaud D, Elez E, Pishvaian MJ, Rugo HS, et al. Safety and Antitumor Activity of Pembrolizumab in Advanced Programmed Death Ligand 1-Positive Endometrial Cancer: Results From the KEYNOTE-028 Study. J Clin Oncol [Internet]. 2017;35(22):2535-2541. doi: https://doi.org/10.1200/ JCO.2017.72.5952

47. Mehnert JM, Panda A, Zhong H, Hirshfield K, Damare $\mathrm{S}$, Lane $\mathrm{K}$, et al. Immune activation and response to pembrolizumab in POLE-mutant endometrial cancer. J Clin Invest [Internet]. 2016;126(6):2334-40. doi: https://doi.org/10.1172/JCI84940

48. Santin AD, Bellone S, Buza N, Choi J, Schwartz PE, Schlessinger J, et al. Regression of ChemotherapyResistant Polymerase $\varepsilon$ (POLE) Ultra-Mutated and MSH6 Hyper-Mutated Endometrial Tumors with Nivolumab. Clin Cancer Res [Internet]. 2016;22(23):5682-5687. doi: https://doi. org/10.1158/1078-0432.CCR-16-1031 